\title{
GENERALIZED IDEAL CONVERGENCE IN PROBABILISTIC NORMED SPACES
}

\author{
BIPAN HAZARIKA
}

Abstract. The aim of this paper is to introduce and study the notion of $I_{\lambda}$-convergence in probabilistic normed space as a variant of the notion of ideal convergence. Also $I_{\lambda}$-limit point and $I_{\lambda}$-cluster point hase been defined and the relation between them have been established. Finally, we establish example which shows that our method of convergence on probabilistic normed space is more general.

Mathematics subject classification (2010): 40G15, 46S70, 54E70.

Keywords and phrases: Ideal convergence, probabilistic normed space, $\lambda$-convergence.

\section{REFERENCES}

[1] C. Alsina, B. Schweizer, A. Sklar, On the definition of a probabilistic normed space, Aequationes Math., 46, 91-98 (1993).

[2] R. C. BuCK, The measure theoretic approach to density, Amer. J. Math. 68 (1946) 560-580.

[3] A. Caserta, G. Di Maio, Lu. D. R. KočinaC, Statistical convergence in function spaces, Abstr. Appl. Anal. Vol. 2011 (2011), Article ID 420419, 11 pages.

[4] J. CONNOR, The statistical and strong p-Cesáro convergence of sequences, Analysis 8 (1988) $47-63$.

[5] K. Dems, On I-Cauchy sequences, Real Anal. Exchange, 30 (1) (2004/2005), 123-128.

[6] H. FAST, Sur la convergence statistique, Colloq. Math. 2 (1951) 241-244.

[7] J. A. FRIDY, On statistical convergence, Analysis, 5 (1985) 301-313.

[8] B. L. Guillén, J. A. R. Lallena, C. Sempi, A study of boundedness in probabilistic normed spaces, J. Math. Anal. Appl. 232 (1999), 183-196.

[9] B. L. Guillén, C. SEmPI, Probabilistic norms and convergence of random variables, J. Math. Anal. Appl. 280 (2003), 9-16.

[10] B. HAZARIKA, On generalized difference ideal convergence in random 2-normed spaces, Filomat, 26 (6) (2012), 1265-1274.

[11] S. KARAKUŞ, Statistical convergence on probabilistic normed spaces, Math. Comm. 12 (2007), 1123.

[12] E. P. Klement, R. Mesiar, E. Pap, Triangular Norms, Kluwer, Dordrecht, 2000.

[13] P. Kostyrko, T. Šalát, W. Wilczyński, I-convergence, Real Anal. Exchange 26 (2) (20002001), 669-686.

[14] P. Kostyrko, M. MaČAj, T. Šalát, M. Sleziak, I-convergence and Extremal I-limit points, Math. Slovaca 2005, 55, 443-64.

[15] K. KumAR, V. KUMAR, On the I and $I^{*}$-Cauchy sequences in probabilistic normed spaces, Mathematical Sciences, 2 (1), 47-58 (2008).

[16] V. Kumar, B. L. Guillén, On Ideal Convergence of Double Sequences in Probabilistic Normed Spaces, Acta Math. Sinica, English Series, 28 (8) (2012), 1689-1700.

[17] B. K. LAHIRI, P. DAS, I and $I^{*}$-convergence in topological spaces, Math. Bohemica, 130 (2005), $153-160$.

[18] B. Lafuerza-Guillén, J. A. Rodríguez-Lallena, C. Sempi, Some classes of probabilistic normed spaces, Rend. Mat., 17 (1997), 237-252.

[19] L. LEINDLER, Über die de la Vallee-Pousinsche Summeierbarkeit allgemeiner Orthogonalreihen, Acta Math. Acad. Sci. Hungar., 16 (1965), 375-387. 
[20] I. J. Maddox, Statistical convergence in a locally convex spaces, Math. Proc. Cambridge Philos. Soc., 104 (1) (1988), 141-145.

[21] G. Di MaIo, LJ. D. R. KočInAC, Statistical convergence in topology, Topology Appl. 156, (2008), $28-45$.

[22] K. Menger, Statistical metrics, Proc. Nat. Acad. Sci. USA, 28 (1942), 535-537.

[23] M. Mursaleen, S. A. Mohiuddine, On ideal convergence in probabilistic normed spaces, Math. Slovaca, 62 (1) (2012), 49-62.

[24] M. R. S. Rahmat, Ideal Convergence on Probabilistic Normed Spaces, Inter. Jour. Stat. Econ., 3 (9) (2009), 67-75.

[25] T. ŠALÁT, On statistical convergence of real numbers, Math. Slovaca, 30 (1980), 139-150.

[26] T. Šalát, B. C. Tripathy, M. Ziman, On some properties of I-convergence, Tatra Mt. Math. Publ. 2004, 28, 279-86.

[27] B. Schweizer, A. Sklar, Statistical metric spaces, Pacific J. Math. 10 (1960), 313-334.

[28] B. Schweizer, A. Sklar, Probabilistic Metric Spaces, North Holland, New York-AmsterdamOxford, 1983.

[29] A. N. ŠERSTNeV, Random normed spaces: Problems of completeness, Kazan Gos. Univ. Ucen. Zap., 122, 3-20 (1962).

[30] H. SteInHAus, Sur la convergence ordinaire et la convergence asymptotique, Colloq. Math. 2 (1951), 73-74.

[31] B. C. TRIPATHY, B. HAZARIKA, I-monotonic and I-convergent sequences, Kyungpook Math. J. 51 (2011), 233-239, DOI 10.5666/KMJ.2011.51.2.233. 